\title{
The mRNA vaccine revolution is the dividend from decades of basic science research
}

$\mathbf{T}$ he 2021 Lasker-DeBakey Clinical Medical Research Award honors Drs. Katalin Karikó and Drew Weissman for key contributions that allowed mRNA vaccines to become reality. The utility and effectiveness of this approach were amply demonstrated in the rapid rollout of vaccines for the prevention of COVID-19. Within a year of their deployment, mRNA vaccines have had a tremendous impact in vaccinated populations in reducing COVID-19 cases and associated mortality. mRNA vaccines are not only a very powerful weapon for containing SARS-CoV-2, but also promise to transform future vaccine approaches, and their development constitutes a true revolution in medicine.

\section{A historical framework for vaccine development}

The practice of vaccination to prevent disease has a long and successful history dating back centuries to use of variolation for prevention of smallpox, and vaccines have been among the most successful interventions for preventing infectious diseases; this strategy has relied on attenuated microbial strains or their component parts for immunization (1). mRNA vaccines represent a departure from prior vaccine strategies because they use nucleic acids to induce the production of microbial antigens in tissue and thus represent a fundamentally different vaccination approach from classical vaccines, which elicit immune responses by administering preformed antigens. The mRNA vaccines are a direct product of the molecular biology revolution that began in the mid-20th century following the discoveries that nucleic acids conferred inheritance (2) and, a decade later, of the structure of DNA (3). In the years that followed, there was an explosion of technologies that allowed the replication of nucleic acids and expression of proteins in laboratory conditions. In 1986, the first recombinant vaccine pro- duced by expressing the surface antigen of hepatitis B was licensed (1). The demonstration in 1989 that exogenous mRNA coding for luciferase could be delivered into a cell line using liposomes, resulting in enzyme expression, was a key discovery on the road to mRNA vaccines (4). For excellent reviews on the development of mRNA vaccines interested readers are referred to previous publications $(5,6)$.

To put the contributions of Drs. Karikó and Weissman in perspective, it is necessary to revisit the intersection among immunology, viral pathogenesis, and molecular biology, where the development of mRNA vaccines faced considerable hurdles. For mRNA vaccines to work, the nucleic acids must get into host cells, where they are translated to express the protein antigen (5). The problem of cell penetration was solved by packaging the mRNA molecules in a hollow lipid sphere known as a liposome that also protects it from degradation by ubiquitous RNAases (5). However, the immune system is very sensitive to RNA because many viruses, such as the coronaviruses, have RNA genomes, and its presence inside cells can be a sign of sign of viral infection. Host immune detection of RNA is mediated by Toll-like receptors, which trigger a cellular response that can shut down protein synthesis and degrade the nucleic acid, events that would defeat the mechanism by which RNA vaccines work. Hence, making mRNA vaccines work required finding a way around the immune system surveillance mechanisms for RNA to avoid triggering an immune response.

\section{Key contributions of Karikó and Weissman}

In 2005, Drs. Karikó and Weissman reported that RNA made with modified nucleosides such as pseudouridine did not activate Toll-like receptors to trigger an inflammatory response (7). This sem- inal discovery provided a solution to the problem of RNA immune recognition and activation and enabled the realization of the concept of mRNA vaccines. In subsequent years, they carried out experiments demonstrating that incorporation of pseudouridine into mRNA not only reduced its immune stimulating properties, but also increased the stability of the nucleic acid, attributes that in combination allowed expression of coded protein and made for an effective vaccine (8). The stage was now set for the clinical development of mRNA vaccines.

\section{Additional fields contributing to mRNA vaccine development}

It is worthwhile to reflect on the broad foundation of knowledge needed to develop mRNA vaccines. The successful effort to create mRNA vaccines required contributions from very different spheres of scientific endeavor. Although all scientific progress involves a complex web of interconnection between different fields, the development of mRNA vaccines has at least four distinct lines of ancestry that can be discerned, from the fields of molecular biology, lipid chemistry, microbiology, and immunology, each of which made critical contributions to their development (Figure 1). In this genealogy, the contributions of Drs. Karikó and Weissman clearly span multiple fields (mRNA chemistry, immunology, vaccines), but they probably fit best within the immunological line of descent (Figure 1). Many of the landmark discoveries in the scientific ancestry of mRNA vaccines have been recognized by Nobel Prizes, but others have not. Many of the Nobel Prizes in the scientific ancestry of mRNA vaccines are in fields of molecular biology and immunology, consistent with the penchant for that honor to recognize basic science contributions (9).

Whereas nucleic acids get most of the glory, perhaps the most underrated contribution to the successful development of mRNA vaccines is the development of liposome and nanoparticle technology, which dates its origin to the description 


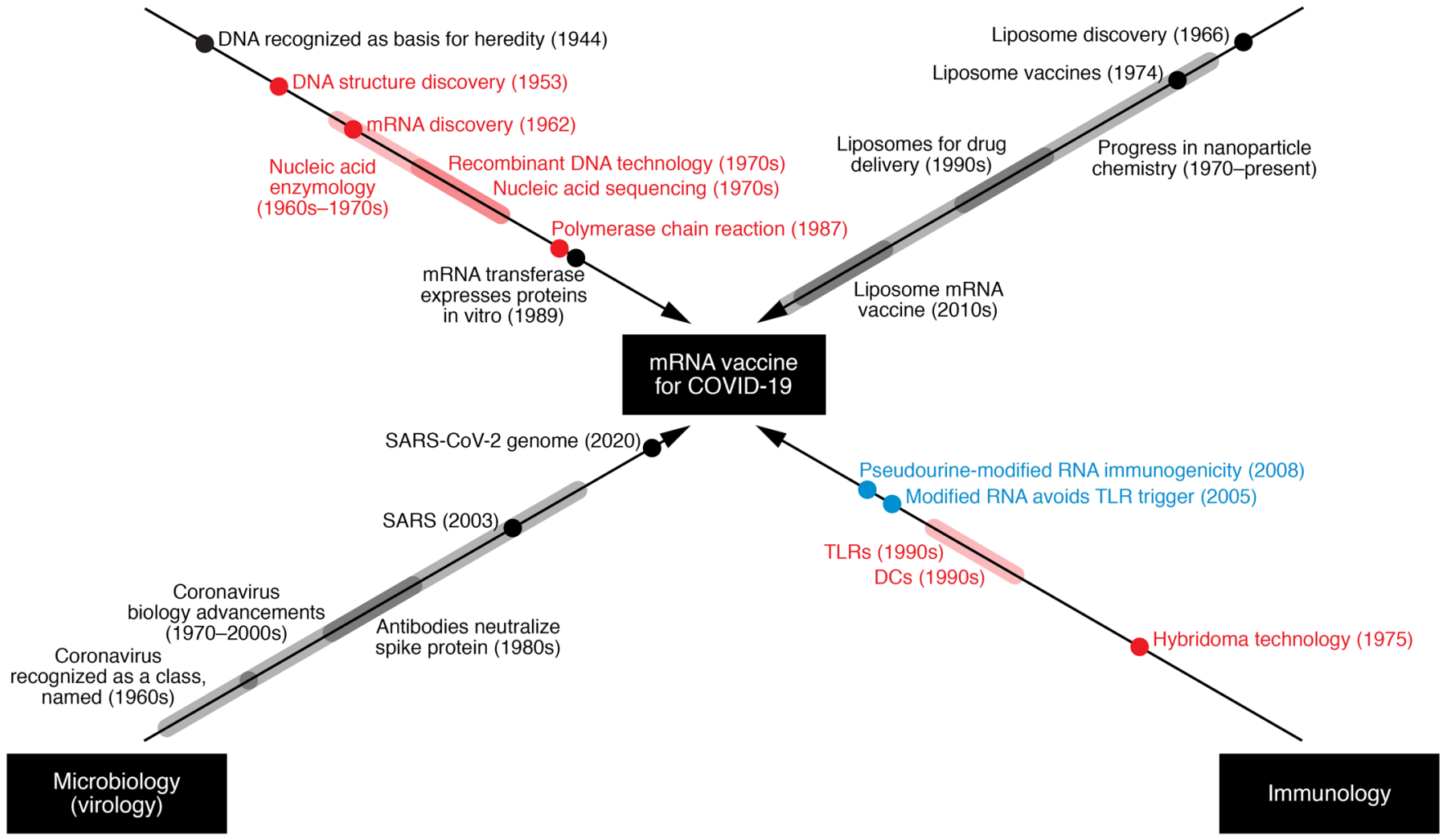

Figure 1. Four major lines of research converge to produce mRNA vaccines against COVID-19. Major discoveries are highlighted (not a complete list). Discoveries recognized with a Nobel Prize are in red font. The contributions of Drs. Karikó and Weissman and their collaborators are highlighted in blue font.

of membrane vesicles. In 1965, a seminal paper showed that phospholipids self-assembled into structures delimited by double-layered membranes that could encapsulate cations (10). The development of liposome delivery systems suitable for mRNA took decades of hard experimental work before these approaches were validated as drug-delivery vehicles in the 1990s (11). The encapsulation of mRNA in lipid vesicles prevents their degradation and allows intracellular delivery, without which mRNA vaccines would not be feasible. Other unsung contributions include knowledge about RNA chemistry and nucleic acid enzymology gathered over decades that provided key knowledge for RNA modification.

The development of mRNA vaccines for SARS-CoV-2 also relied on a large body of knowledge amassed by the virology community on coronavirus biology and immunology. Some of the earliest work showing that antibodies to the coronavirus spike protein were neutralizing was done with an avian coronavirus. In the 1980s, monoclonal antibodies (mAbs), which are a product of the Nobel Prize-winning discovery of hybridoma technology, were used to show that binding to epitopes in the hypervariable region of the avian coronavirus IBD spike glycoprotein could neutralize the virus $(12,13)$. Furthermore, those studies showed that single amino acid changes to the spike protein could abrogate mAb-neutralizing capacity (12), anticipating the rise of SARS-CoV-2 variants that reduced the efficacy of $\mathrm{mAb}$ COVID-19 therapies (14). The SARS outbreak of 2003 that was followed by MERS established the threat of coronaviruses to human populations and stimulated a tremendous amount of research that was available when COVID-19 struck in 2019.

Prior to COVID-19, most developmental work with mRNA vaccines was done with cancer (5), as mRNA vaccines brought the promise of great specificity, which was needed to elicit responses to rare mutated genes in malignant tissues, but spare nor- mal tissue. In terms of infectious diseases, some development work was done with mRNA vaccines against HIV and influenza virus, viruses that are notorious for their antigenic variability, which has precluded making a successful vaccine in the case of the former and requires yearly vaccinations in the case of the latter. The first mRNA vaccine against an infectious disease that was evaluated in humans targeted rabies virus, and a clinical trial showed that it elicited functional antibodies (15).

\section{A transformative event for humanity}

The success of mRNA vaccines against SARS-CoV-2 implies that this technology can be applied to target any pathogen for which a protein can be identified as an antigen that elicits protective immunity. mRNA vaccines represent a potentially disruptive technology for the vaccine industry. This approach could make some currently used vaccines obsolete, such as the influenza vaccines, which require 
guessing the likely virus variant and production timetables that do not allow flexibility, and potentially allow the rapid development of new vaccines against microbes for which it has been difficult to make vaccines, such as HIV. In surveying currently available vaccines, all antiviral vaccines trigger immune responses to proteins from inactivated or attenuated viruses or their components, which mRNA vaccines can readily be designed to produce. For bacterial diseases, our most successful vaccines are toxoids and polysaccharide-protein conjugates, neither of which is easily reproduced with mRNA vaccines, since toxoids are new antigens created by denaturing toxins, while conjugate vaccines require a complex choreography for antigen recognition. However, it is possible that the success of mRNA vaccines will stimulate a new search for bacterial, fungal, and parasite protein antigens that elicit protective immune responses.

The mRNA vaccine story shows the huge benefits that society can reap from investing in basic science. Almost certainly, no investigator or observer of science in the past could have predicted that any one of the thousands of papers describing what needed to be known, from RNA to lipid chemistry to cell biology to immunology to virus structure, to cite just a few strands of the necessary knowledge base, would one day allow humanity to respond so rapidly to a new viral threat. In the first 21 years of the 21st century, humanity has faced at least six major viral outbreaks, in the form of SARS, MERS, Ebola, Zika, influenza, and SARS-CoV-2. Beyond infectious disease threats, additional calamities in the form of climate change, ecological degradation, food supply uncertainty, and social instability suggest that humanity faces rough years ahead. Whereas each of these challenges requires different solutions, the common thread is that knowledge gained from fundamental research can give humanity new options for meeting existential threats and that knowledge must be broad based and gained through painstaking scientific work. Continued investment in basic science is humanity's best insurance policy.

\section{Arturo Casadevall Deputy Editor}

1. Plotkin S. History of vaccination. Proc Natl Acad Sci US A. 2014;111(34):12283-12287.

2. Avery OT, et al. Studies on the chemical nature of the substance inducing transformation of pneumococcal types: induction of transformation by a desoxyribonucleic acid fraction isolated from pneumococcus type III. J Exp Med. 1944;79(2):137-158.

3. Watson JD, Crick FH. Molecular structure of nucleic acids; a structure for deoxyribose nucleic acid. Nature. 1953;171(4356):737-738.

4. Malone RW, et al. Cationic liposome-mediated RNA transfection. Proc Natl Acad Sci US A.
1989;86(16):6077-6081.

5. Pardi N, et al. mRNA vaccines - a new era in vaccinology. Nat Rev Drug Discov. 2018;17(4):261-279.

6. Verbeke R, et al. Three decades of messenger RNA vaccine development. Nano Today. 2019;28:100766

7. Karikó K, et al. Suppression of RNA recognition by Toll-like receptors: the impact of nucleoside modification and the evolutionary origin of RNA. Immunity. 2005;23(2):165-175.

8. Karikó K, et al. Incorporation of pseudouridine into mRNA yields superior nonimmunogenic vector with increased translational capacity and biological stability. Mol Ther. 2008;16(11):1833-1840.

9. Casadevall A, Fang FC. Is the Nobel Prize good for science? FASEB J. 2013;27(12):4682-4690.

10. Bangham AD, et al. Diffusion of univalent ions across the lamellae of swollen phospholipids. JMol Biol. 1965;13(1):238-252.

11. Gregoriadis G. Liposomes in drug delivery: how it all happened. Pharmaceutics. 2016;8(2):19.

12. Cavanagh D, et al. Amino acids within hypervariable region 1 of avian coronavirus IBV (Massachusetts serotype) spike glycoprotein are associated with neutralization epitopes. Virus Res. 1988;11(2):141-150.

13. Mockett AP, et al. Monoclonal antibodies to the S1 spike and membrane proteins of avian infectious bronchitis coronavirus strain Massachusetts M41. J Gen Virol. 1984;65(Pt 12):2281-2286.

14. Widera $\mathrm{M}$, et al. Limited neutralization of authentic SARS-CoV-2 variants carrying E484K in vitro [published online July 5, 2021]. J infect Dis. https://doi.org/10.1093/infdis/jiab355.

15. Alberer M, et al. Safety and immunogenicity of a mRNA rabies vaccine in healthy adults: an open-label, non-randomised, prospective, first-in-human phase 1 clinical trial. Lancet 2017;390(10101):1511-1520 